Case Report

\title{
Primary Mucinous Adenocarcinoma of the Vermilorm Appendix with High Grade Microsatellite Instability
}

\section{Moritz Komm ${ }^{1 凶}$, Michaela Kronawitter-Fes| ${ }^{2}$, Marcus Kremer², Ludwig Lutz ${ }^{3}$, Elke Holinski-Feder ${ }^{4}$, Reinhard Kopp ${ }^{1}$}

1. Department of General Surgery, Klinikum München-Harlaching, Munich, Germany

2. Department of Pathology, Klinikum München-Harlaching, Munich, Germany

3. Department of Haematology and Oncology, Klinikum München-Harlaching, Munich, Germany

4. Medical Genetic Center, Munich, Germany

Corresponding author: Moritz Komm, M.D., Klinik für Allgemein-, Viszeral-, Gefäß- und Thoraxchirurgie, Städtisches Klinikum München-Harlaching, Sanatoriumsplatz 2, 81545 München, Germany, e-mail: moritz@dr-komm.de, Phone: +49 89 62102302

(C) Ivyspring International Publisher. This is an open-access article distributed under the terms of the Creative Commons License (http://creativecommons.org/ licenses/by-nc-nd/3.0/). Reproduction is permitted for personal, noncommercial use, provided that the article is in whole, unmodified, and properly cited.

Received: 2010.12.18; Accepted: 2011.05.13; Published: 2011.05.23

\begin{abstract}
Primary adenocarcinoma of the vermiform appendix is a rare entity and is frequently discovered by the pathologist following appendectomy for suspected appendicitis.

We present a 42-year-old male with primary mucinous adenocarcinoma of the appendix initially presenting symptoms of acute appendicitis. Histological investigation of the appendectomy specimen showed a mucinous adenocarcinoma and the patient was treated by secondary right hemicolectomy giving the final histopathological classification of an UICC IIIC tumor. Since the patient fulfills the revised Bethesda criteria analysis of immunoreactivity of DNA mismatch repair proteins was performed showing loss of MLH1 and MSH2 expression associated with high microsatellite instability (MSI-H), not yet reported for primary mucinous appendiceal carcinoma. Further genetic analysis for DNA mismatch repair gene mutations were negative. The patient received intensified adjuvant chemotherapy according to the FOLFOX-4-scheme, since MSI-H colorectal carcinomas might show lower response rates following standard 5-FU-based adjuvant chemotherapy.
\end{abstract}

Key words: Appendixcarcinoma, microsatellite instability, mucinous appendixcarcinoma, DNA mismatch repair genes

\section{Introduction}

Primary adenocarcinoma of the vermiform appendix is a rare entity and is frequently discovered by the pathologist following appendectomy for suspected appendicitis. Neoplasms of the appendix are found in $1 \%$ of all appendectomy specimen with a frequency of appendiceal adenocarcinoma in $0,1 \%$ of the specimen investigated. The incidence of that type of neoplasm varies from 0,01 to 0,2 per 100.000 per- sons per year (1) and it constitutes less than $0,5 \%$ of all gastrointestinal malignant neoplasms $(2,3,4)$.

\section{Case report}

We present a 42 year old male with mucinous adenocarcinoma of the appendix who initially presented with typical symptoms of acute appendicitis. Furthermore the patient reported recurrent peranal 
loss of blood, which was suspected to be associated with a history of colitis ulcerosa, known since 1997 and continuously treated by NSAID.

The patient was treated by an uncomplicated laparoscopic appendectomy. The histopathological examination of the specimen surprisingly revealed a pT4a (local infiltration of the visceral peritoneum) mucinous adenocarcinoma of the appendix (figure 1) with positive resection margins (R1 resection). Staging examinations including colonoscopy, gastroscopy, thoracical x-ray and abdominal ultrasound were normal. Therefore, right hemicolectomy according to the principals of oncologic surgery was performed. The final histopathological classification described a pT4a, pN2 (6/15), L1, V0, M0, G3, R0 tumor, corresponding to UICC IIIC classification.

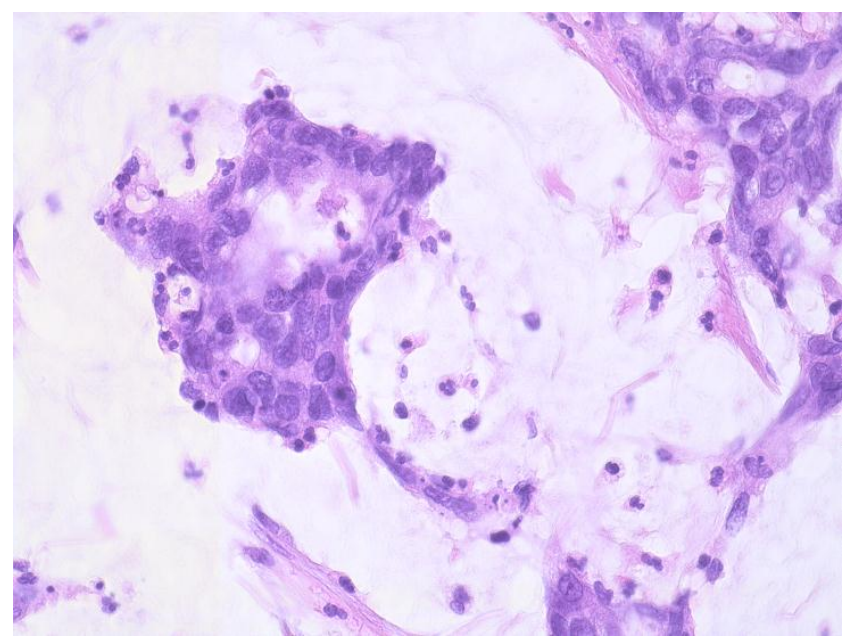

Figure 1: Haematoxylin und eosin staining of the appendix carcinoma. The mucinous lakes constitute more than $50 \%$ of the tumor mass which is a criterion for the mucinous type of the appendiceal carcinoma.

Because of the young age of the patient hereditary cancer syndrome was considered, although the family tumor history was negative for gastrointestinal tumors or other malignancies.

Immunhistochemical examination showed a lack of expression of the two mismatch repair genes MLH1 (figure 2) and MSH2 (figure 3) possibly associated with hereditary non-polyposis colon cancer syndrome (HNPCC or Lynch syndrome). Analysis of microsatellite instability (MSI) showed high grade MSI (MSI-H) testing the markers BAT25 (stable), BAT 26 (stable), D2S123 (instable), D5S346 (instable) and D17S250 (instable) (figure 4). No mutation in the MLH1- and MSH2-genes was detected by gene se- quencing. In addition, the tumor sample was negative for B-Raf mutation or evidence of DNA hypermethylation.

Due to the histopathological classification intensified adjuvant chemotherapy according to the FOLFOX 4 scheme was started five weeks after surgery. Currently after 24 month of follow up the patient is without detectable tumor recurrence, as shown by abdominal MR imaging, colonoscopy and ultrasound examination.

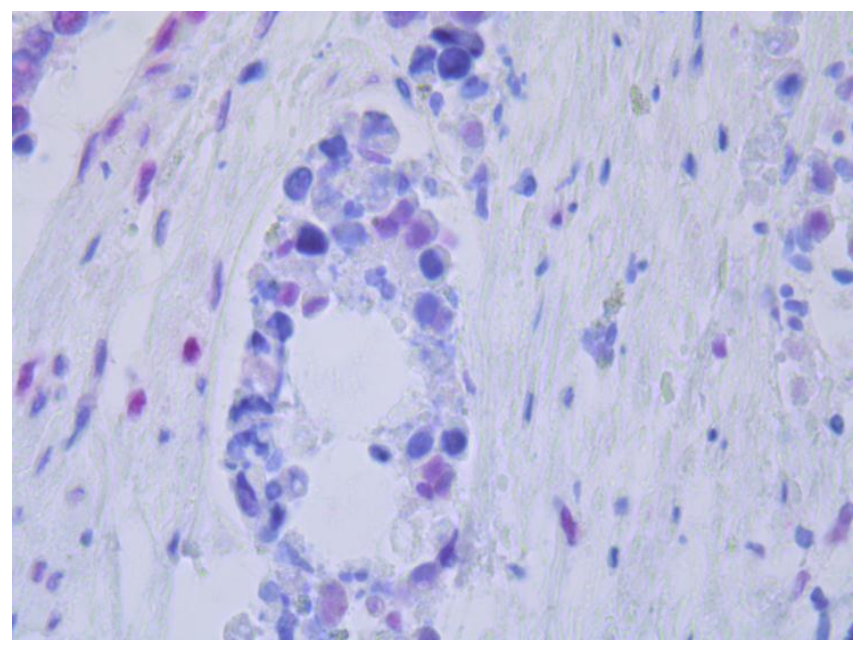

Figure 2: Immunhistochemical staining of the tumor. The blue colored cores as a sign of loss of expression of MLH1. The red colored cores show normal expression of MLH1.

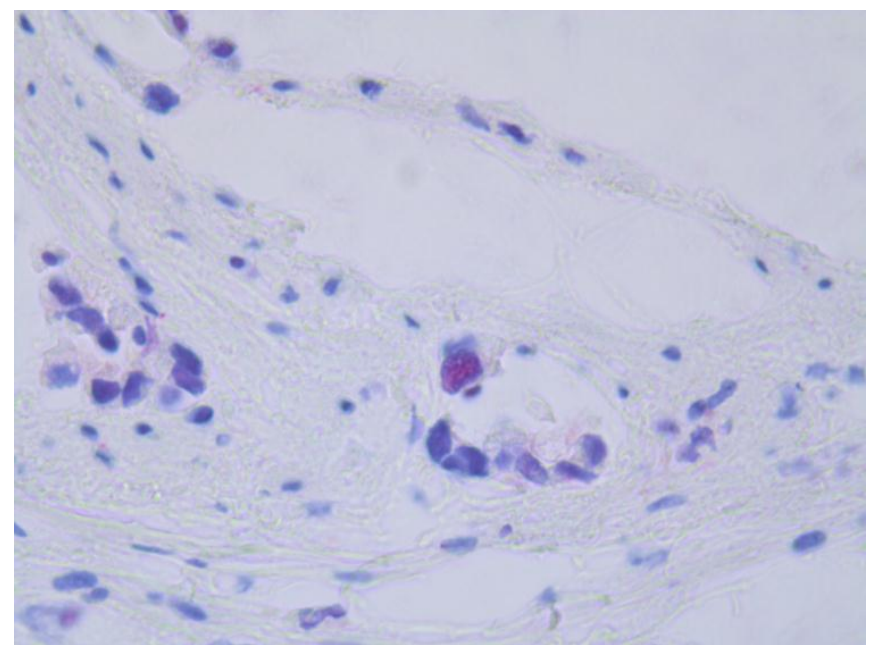

Figure 3: Immunhistochemical staining of the tumor. The blue colored cores as a sign of loss of expression of $M S H 2$. The red colored core in the center shows normal expression of MSH2. 
(A)D2S123 (nstable)

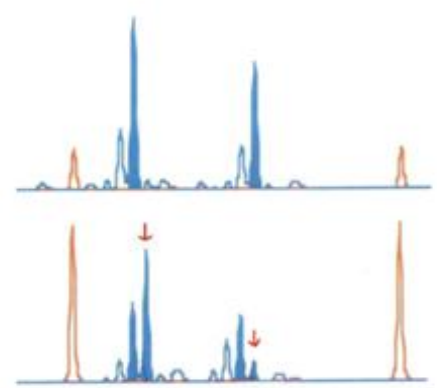

(B) DSS346 (mstable)

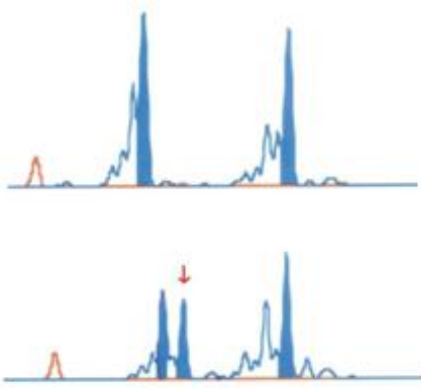

(C) D17S250 (instable)

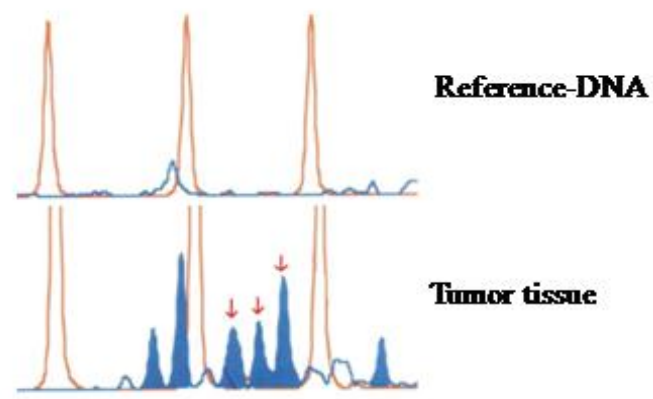

Figure 4: PCR amplification of the repetitive DNA sequences D2S123 (A), D5S346 (B) and D17S250 (C). The upper sequences show the traces of the reference-DNA gained from peripheral blood. The lower sequences show the traces of the tumor tissue. Differences are marked by red arrows.

\section{Discussion}

Primary adenocarcinoma of the appendix is a rare neoplasm detected in less than $0.5 \%$ of all neoplasms of gastrointestinal origin. Carcinomas of the appendix are usually classified according to histopathological examination into four distinct subtypes with varying frequencies: colonic type adenocarcinoma, mucinous adenocarcinoma, signet ring type adenocarcinoma and others. In a group of 1095 patients with primary appendiceal adenocarcinoma McCusker et al. (6) found 56,0\% of tumors classified as mucinous appendix carcinoma, $37,6 \%$ were belonging to the colonic and $6,4 \%$ to the signet ring cell subtype. The five year survival rate for all patients was $54.4 \%$ and was associated with the stage of disease, the grading and the histological subtype. In a group of 94 patients Nitecki et al. (7) found a survival of $100 \%$ for patients with tumors classified as Dukes A, $67 \%$ for Dukes B, $50 \%$ for Dukes C and $6 \%$ for Dukes D carcinomas. The data presented in the literature concerning the prognosis according to the different histological subtypes is not yet conclusive. McCusker et al. (6) reported 5-year survival rates of $44 \%$ for tumors with the mucinous subtype, $52 \%$ for the colonic subtype and $20 \%$ for the signet ring cell subtype. Other authors report that the mucinous histology is the best prognostic factor $(3,7,8)$. The general prognosis seems to be close to that reported for colonic cancers $(6,7,9)$.

While simple appendectomy seems to be sufficient for pT1 carcinoma of the appendix most tumors present as advanced invasive carcinomas and secondary right hemicolectomy is usually recommended as the operative treatment of choice (8). Malignant infiltration of locoregional lymph nodes has to be expected in about on third of the patients and especially in advanced invasive tumors. Adjuvant chemotherapy is therefore recommended in these patients with lymph node positive or locally advanced tumors in accordance with the recommendation for colon cancer. However, frequently carcinomas of the appendix show local infiltration in the surrounding peritoneum with increased risk of locoregional recurrence. For these patients with peritoneal tumor infiltration or peritoneal metastasis cytoreductive surgery in combination with hyperthermic chemotherapy might be an additional therapeutic option (10).

Studies investigating the tumorbiologic alterations of carcinomas of the appendix have so far reported classic mutations associated with sporadic colorectal cancer including frequent K-ras mutations, rare p53 protein overexpression and all tumors investigated so far were microsatellite stable (5). Therefore, the mucinous appendix carcinoma with high grade microsatellite instability, first described in our report, seems to represent a distinct tumor etiology and might describe a yet unidentified alteration in the DNA repair pathway with possible implications for chemotherapeutic modifications during adjuvant chemotherapy. Microsatellite instabilities are found in tumors associated with genetic defects in DNA mismatch repair, but they are also found in $10-20 \%$ of sporadic advanced colon carcinomas mainly caused by DNA hypermethylation leading to defects in DNA mismatch repair. Lanza et al. (11) detected a loss of expression of MLH1- or MSH2-genes in 90,9\% of MSI-H colon carcinomas, whereas all MSI-L (low instability) and MSS (stable) tumors showed normal expression of both proteins (12). There is a considerable number of obviously HNPCC associated tumors in which a gene defect cannot be found. Genomic rearrangements, not detectable by PCR or sequencing, were found in a considerable proportion of HNPCC 
families without point mutations (13). Grabowski et al. (14) found in their series of 64 patients without detectable point mutations in $17 \%$ pathogenic alterations in MLH1 and MSH2 genes caused by deletions. As our patient showed lack of protein expression of two mismatch repair genes, microsatellite instability and furthermore fulfilled the revised Bethesda Criteria, a yet unidentified genetic defect, associated with defects in mismatch repair might represent a cofactor for the development of the described mucinous appendiceal carcinoma.

However, although prognosis and survival seems to be better for colon cancer patients with microsatellite instable tumors, microsatellite instability was associated with reduced responsiveness on 5-FU based adjuvant chemotherapy in other reports (15). Therefore, microsatellite instability has to be considered during adjuvant chemotherapy for colorectal and also for rare cases of appendiceal carcinomas in patients with early onset tumors and limited response or tumor progression during initial 5-FU based chemotherapy. Although the consideration of microsatellite instability is increasing, responsiveness on 5-FU-based first line chemotherapy is temporized in treatment of microsatellite instable colon or appendix carcinoma. In case of tumor progression or non-response the addition of Irinotecan, Oxaliplatin or antitumor biologicals as a part of the adjuvant chemotherapy concept should be considered and is currently investigated in clinical trials.

Therefore, our patient received intensified adjuvant chemotherapy according to FOLFOX-4 scheme in accordance to the treatment of advanced colon cancer.

\section{Conclusions and summary}

The patient reported shows the very rare entity of a mucinous adenocarcinoma of the appendix with high grade microsatellite instability, not yet reported in the literature before. Therefore, tumorbiologic alterations with so far unidentified alterations of the DNA mismatch repair pathway leading to reduced expression of DNA repair proteins and microsatellite instability might be associated with mucinous adenocarcinomas of the appendix. Following 5-FU based adjuvant chemotherapy relevant variability in antitumor response of MSI-H tumors and therefore, intensified chemotherapy protocols will have to be considered in these usually young patients.

\section{Contributions}

Moritz Komm: responsible for treatment of patient, writing of the article; Michaela Kronawitter-Fesl: pathological examinations; Marcus Kremer: pathological examinations; Ludwig Lutz: oncologic con- sultant responsible for the adjuvant oncologic therapy; Elke Holinski-Feder: genetic examinations; Reinhard Kopp: responsible for treatment of patient, writing of the article.

\section{Conflict of Interest}

The authors have declared that no conflict of interest exists.

\section{References}

1. Rassu PC, Cassinelli G, Ronzitti F, Bronzino P, Stanizzi T, Casaccia M. Primary adenocarcinoma of the appendix. Case report and review of the literature. Min Chir. 2002; 57(5):695-8.

2. O'Donnell ME, Badger SA, Beattie GC, Carson J, Garstin WI. Malignant neoplasms of the appendix. Int J Colorectal Dis. 2007; 22:1239-48.

3. McGory ML, Maggard MA, Kang H, O'Connell JB, Ko CY. Malignancies of the appendix: Beyond case series reports. Dis Colon Rectum. 2005; 48:2264-71.

4. Ko YH, Jung CK, Oh SN, Kim TH, Won HS, Kang JH, Kim HJ, Kang WK, Oh ST, Hong YS. Primary signet ring cell carcinoma of the appendix: A rare case report and our 18-year experience. World J Gastroenterol. 2004; 14(37):5763-8.

5. Kabbani W, Houlihan PS, Luthra R, Hamilton SR, Rashid A. Mucinous and nonmucinous appendiceal adenocarcinomas: different clinicopathological features but similar genetic alterations. Mod Pathol. 2002; 15(6):599-605.

6. McCusker ME, Cote TR, Clegg LX, Sobin LH. Primary malignant neoplasms of the appendix: A data population-based study from the surveillance, epidemiology and end-results program, 1973-1998. Cancer. 2002; 94:3307-12.

7. Nitecki SS, Wolff BG, Schlinkert R, Sarr MG. The natural history of surgically treated primary adenocarcinoma of the appendix. Ann Surg. 1994; 219(1):51-7.

8. Cortina R, McCormick J, Kolm P, Perry RR. Management and prognosis of adenocarcinoma of the appendix. Dis Colon Rectum. 1995; 38(8):848-52.

9. Ito H, Osteen RT, Bleday R, Zinner MJ, Ashley SW, Whang EE. Appendiceal adenocarcinoma: Long-term outcomes after surgical therapy. Dis Colon Rectum. 2004; 47:474-80.

10. Marcotte E, Sideris L, Drolet P, Mitchell A, Frenette S, Leblanc G, Leclerc YE, Dube P. Hyperthermic intraperitoneal chemotherapy with oxaliplatin for peritoneal carcinomatosis arising from appendix: preliminary results of survival analysis. Ann Surg Oncol. 2008; 15(10):2701-2708.

11. Lanza G, Gafà R, Maestri I, Santini A, Matteuzzi M, Cavazzini L. Immunhistochemical pattern of MLH1/MSH2 expression is related to clinical and pathological features in colorectal adenocarcinomas with microsatellite instability. Mod Pathol. 2002; 15(7):741-9.

12. Schiemann U, Müller-Koch $Y$, Gross M, Daum J, Lohse $P$, Baretton G, Muders M, Mussack T, Kopp R, Holinsky-Feder E. Extended microsatellite analysis in microsatellite stable, MSH2 and MLH1 mutation-negative HNPCC patients: genetic reclassification and correlation with clinical features. Digestion. 2004; 69(3):166-76.

13. Pistorius S, Görgens H, Plaschke J, Hoehl R, Krüger S, Engel C, Saeger HD, Schackert HK. Genomic rearrangements in MSH2, MLH1 or MSH6 are rare in HNPCC patients carrying point mutations. Cancer Lett. 2007; 258(1):89-95

14. Grabowski M, Mueller-Koch Y, Grasbon-Frodl E, Koehler U, Keller G, Vogelsang H, Dietmaier W, Kopp R, Siebers U, Schmitt W, Neitzel B, Gruber M, Doerner C, Kerker B, Ruemmele P, Henke G, Holinnkski-Feder E. Deletions account for $17 \%$ of pathogenetic germline alterations in MLH1 and 
MSH2 in hereditary nonpolyposis colorectal cancer (HNPCC) families. Genet Test. 2005; 9(2):138-46

15. Benatti P, Gafà R, Barana D, Marino M, Scarselli A, Pedroni M, Maestri I, Guerzoni L, Roncucci L, Menigatti M, Roncari B, Maffei S, Rossi G, Ponti G, Santini A, Losi L, Di Gregorio C, Oliani C, Ponz de Leon M, Lanza G. Microsatellite instability and colorectal cancer prognosis. Clin Cancer Res. 2005; 11(23):8332-40 